\title{
Knowledge, experiences and attitudes concerning genetics among retinoblastoma survivors and parents
}

\author{
Jessica A Hill' ${ }^{1,7} \cdot$ Amal Gedleh $^{1,2} \cdot$ Siwon Lee $^{1,2} \cdot$ Kaitlyn A Hougham $^{1} \cdot$ Helen Dimaras ${ }^{1,3,4,5,6}$
}

Received: 13 April 2017 / Revised: 23 August 2017 / Accepted: 10 October 2017 / Published online: 29 January 2018

(c) European Society of Human Genetics 2018

\begin{abstract}
Clinical genetic services are increasingly providing a more nuanced understanding of genetic disease diagnostics and future risk for patients. Effectively conveying genetic information is essential for patients to make informed decisions. This is especially important for survivors of heritable cancers such as retinoblastoma (childhood eye cancer), where survivors who carry a germline mutation in the $R B 1$ gene are at increased risk of second cancers in adulthood, and of passing on the disease risk to future offspring. We conducted focus groups with adult survivors of retinoblastoma and parents of children with retinoblastoma, to uncover their knowledge of, experiences with and attitudes about retinoblastoma genetics and related impacts of the cancer. Results revealed that participants understood that retinoblastoma was a genetic disease, but often misunderstood the implications of genetics on cancer phenotype and risk. Experiences with genetic testing and counseling were generally positive, however, participants reported challenges in accessing genetic information and psychosocial support. Participants suggested more educational resources, peer-to-peer counseling, and psychosocial support would enhance uptake of important genetic information. The results of the study will inform patient-oriented approaches to deliver comprehensive genetic healthcare.
\end{abstract}

Amal Gedleh and Siwon Lee contributed equally to this work.

Electronic supplementary material The online version of this article (https://doi.org/10.1038/s41431-017-0027-9) contains supplementary material, which is available to authorized users.

Helen Dimaras

helen.dimaras@utoronto.ca

1 Department of Ophthalmology and Vision Sciences, The Hospital for Sick Children, Toronto, Canada

2 Department of Health Promotion, Dalla Lana School of Public Health, University of Toronto, Toronto, Canada

3 Division of Clinical Public Health, Dalla Lana School of Public Health, University of Toronto, Toronto, Canada

4 Department of Ophthalmology and Vision Sciences, Faculty of Medicine, University of Toronto, Toronto, Canada

5 Child Health Evaluative Sciences Program, SickKids Research Institute, Toronto, Canada

6 Department of Human Pathology, College of Health Sciences, University of Nairobi, Nairobi, Kenya

7 Present address: Department of Molecular Genetics, University of Toronto, Toronto, Canada

\section{Introduction}

Genetic testing is integral to diagnosing and managing heritable cancer. Genetic counseling assists patients and families in interpreting and coping with test results. Crucially, effective genetic counseling leads to greater treatment compliance for affected individuals and thus better outcomes, as well as informed family planning and lifestyle decisions, and improved coping with diagnosis of a heritable condition [1,2].

Retinoblastoma was the first cancer for which a genetic origin was identified. It is precipitated by loss-of-function of the tumor-suppressor gene RBI [3], or in rare cases, amplification of $M Y C N$ [4]. Genetic testing and counseling play a critical role in the determination of the heritable nature of a retinoblastoma diagnosis, in addition to identifying cancer risk for patients and related family members [5]. Healthcare management recommendations for patients and patient families with heritable retinoblastoma are significant and distinct from non-heritable cases.

All children with bilateral (both eyes affected) retinoblastoma have the heritable form of cancer, as the predisposing mutation is constitutional [5, 6]. About $15 \%$ of children with unilateral (one eye affected) retinoblastoma 
also have the heritable form, often a result of a "lowpenetrance" constitutional mutation that fails to cause tumors in both eyes [6]. A constitutional RB1 mutation also predisposes to later, second cancers. However, presence of a constitutional mutation does not mean that the mutant allele was inherited; only $5-10 \%$ of retinoblastoma cases are familial, as most cases are caused by a new mutation arising during fetal development. Depending on when during embryogenesis the mutation is acquired, the $R B 1$ mutation can affect a proportion of cells in the body, also known as mosaicism [7]. Genetic testing can identify the genetic cause of retinoblastoma, which can then be used to screen other family members, including parents, siblings, and future offspring of the affected individual [6]. The genetic testing results are then disseminated to families via genetic counseling while the affected children are still young. In Canada, genetic testing and counseling are part of the standard of care for retinoblastoma [8], and accessed by families via their retinoblastoma treatment center. It is unclear how much of this information is understood, prioritized, utilized, or transferred to adult survivors of retinoblastoma.

The goal of this study was to uncover the knowledge of, experiences with and attitudes about retinoblastoma genetics among retinoblastoma survivors and parents of children with retinoblastoma. We hypothesized that these data might identify barriers and facilitators to understanding, uptake and application of genetic information about retinoblastoma. Ultimately, the goal is to minimize healthcare barriers through developing and testing innovative, patient-oriented approaches to deliver comprehensive cancer genetic services.

\section{Subjects and methods}

\section{Study purpose and design}

This qualitative study aimed to gain an in-depth perspective of the knowledge of, experiences with and attitudes about retinoblastoma genetics among adult retinoblastoma survivors and parents of children with retinoblastoma concerning genetics. Research Ethics Board approval was granted by The Hospital for Sick Children (REB \# 1000050339).

\section{Study participant recruitment and selection}

Study participants were recruited with the help of publicly listed retinoblastoma societies or organizations (e.g., the Canadian Retinoblastoma Society) and the retinoblastoma team at The Hospital for Sick Children. Participants were required to meet the following inclusion criteria: (i) 18 years of age or an emancipated minor; and (ii) an adult retinoblastoma survivor or parent/legal guardian of a child with retinoblastoma. All participants provided written informed consent prior to participating in the study.

\section{Demographic questionnaire}

Participants completed a demographic questionnaire that asked about sex, age, primary language, place of residence, ethnicity, religion, education, employment, income, and number of children. The questionnaire also requested details on the retinoblastoma diagnosis, including details of family members affected by retinoblastoma, whether unilateral or bilateral, genetic testing status, heritability, and length of time since diagnosis.

\section{Focus group conduct}

Focus groups were chosen to as a data collection method to facilitate an open-ended discussion more likely to reveal unanticipated insights that may be missed using quantitative methods. All focus groups took place at The Hospital for Sick Children between October and December of 2015. Each focus group discussion lasted between 60 and 90 min and was moderated in English. There were 4-6 participants per group. The moderator used a focus group interview guide developed by the study authors JAH and HD (both with $\mathrm{PhDs}$ in medical genetics and expertise in retinoblastoma). The interview guide asked about: (i) knowledge of retinoblastoma and retinoblastoma genetics, including cause of disease and interpretation of the words "genetic" and "inheritance"; (ii) experience with retinoblastoma genetics, including genetics as part of treatment and followup, sources of information, and communication with the healthcare team; and (iii) attitudes about retinoblastoma genetics, including perceived implications, benefits or challenges of retinoblastoma genetics. After the focus groups, a primer on retinoblastoma genetics was distributed, and the moderator clarified points of confusion or erroneous information that may have arisen during the discussion. Participants were directed to their healthcare providers for any questions specific to their personal experience with retinoblastoma.

\section{Data analysis}

All audio recordings were transcribed verbatim and participants were de-identified; patient ID codes (e.g., O1, N2, D3) are used to distinguish between participants in the results section. QSR International NVivo 11 qualitative data analysis software was used for data management and coding of the transcripts. Inductive thematic analysis was employed to identify common themes in the transcripts. Two researchers coded the data independently. Discrepancies were settled consulting a third member of the research team. 
Table 1 Study participant demographics

\begin{tabular}{|c|c|c|}
\hline & $n$ & $\%$ \\
\hline \multicolumn{3}{|l|}{ Sex } \\
\hline Female & 12 & $80 \%$ \\
\hline Male & 3 & $20 \%$ \\
\hline \multicolumn{3}{|l|}{ Age } \\
\hline $18-25$ & 0 & $0 \%$ \\
\hline $26-40$ & 11 & $73 \%$ \\
\hline $41-55$ & 3 & $20 \%$ \\
\hline $56+$ & 1 & $7 \%$ \\
\hline \multicolumn{3}{|l|}{ Primary language } \\
\hline English & 12 & $80 \%$ \\
\hline French & 0 & $0 \%$ \\
\hline Other & 3 & $20 \%$ \\
\hline \multicolumn{3}{|l|}{ Ethnicity } \\
\hline Caucasian & 10 & $67 \%$ \\
\hline South Asian & 2 & $13 \%$ \\
\hline East Asian & 2 & $13 \%$ \\
\hline African & 1 & $7 \%$ \\
\hline \multicolumn{3}{|l|}{ Religion } \\
\hline Non-religious & 7 & $47 \%$ \\
\hline Christianity & 5 & $33 \%$ \\
\hline Hinduism & 2 & $13 \%$ \\
\hline Judaism & 1 & $7 \%$ \\
\hline \multicolumn{3}{|l|}{ Education } \\
\hline University degree & 5 & $33 \%$ \\
\hline College diploma & 4 & $27 \%$ \\
\hline $\begin{array}{l}\text { Graduate/ } \\
\text { Professional school }\end{array}$ & 4 & $27 \%$ \\
\hline Some high school & 2 & $13 \%$ \\
\hline \multicolumn{3}{|l|}{ Employment } \\
\hline Employed & 12 & $80 \%$ \\
\hline Homemaker & 2 & $13 \%$ \\
\hline Student & 1 & $7 \%$ \\
\hline \multicolumn{3}{|l|}{ Income } \\
\hline$<\$ 25,000 \mathrm{CAD}$ & 1 & $7 \%$ \\
\hline $\begin{array}{l}\$ 25,000-\$ 50,000 \\
\text { CAD }\end{array}$ & 3 & $20 \%$ \\
\hline $\begin{array}{l}\$ 50,000-\$ 75,000 \\
\text { CAD }\end{array}$ & 2 & $13 \%$ \\
\hline $\begin{array}{c}\$ 75,000- \\
\$ 100,000 \mathrm{CAD}\end{array}$ & 3 & $20 \%$ \\
\hline $\begin{array}{l}\text { Over } \$ 100,000 \\
\text { CAD }\end{array}$ & 6 & $40 \%$ \\
\hline \multicolumn{3}{|l|}{ Current residence } \\
\hline Metropolitan city & 9 & $60 \%$ \\
\hline Small town & 5 & $33 \%$ \\
\hline Rural village & 1 & $7 \%$ \\
\hline \multicolumn{3}{|l|}{ \# Of children } \\
\hline 0 & 0 & $0 \%$ \\
\hline
\end{tabular}

Table 1 (continued)

\begin{tabular}{lll}
\hline & $n$ & $\%$ \\
\hline 1 & 6 & $40 \%$ \\
2 & 7 & $47 \%$ \\
$3+$ & 2 & $13 \%$ \\
\hline
\end{tabular}

\section{Results}

\section{Study participant demographics}

Fifteen individuals participated in three focus groups at the Hospital for Sick Children. Participants were mainly female $(12 / 15,80 \%)$, between 26 and 40 years of age $(11 / 15,73 \%)$, spoke primarily English $(12 / 15,80 \%)$, and identified as Caucasian $(10 / 15,67 \%)$ (Table 1). Close to half reported that they were non-religious $(7 / 15,47 \%)$, whereas the remainder indicated affiliation with Christianity (5/15, $33 \%)$, Hinduism $(2 / 15,13 \%)$, and Judaism $(1 / 15,7 \%)$. Most participants had a university or college education, were employed, and reported earning a high income (Table 1). All participants were parents of at least one child.

\section{Study participant relationship with retinoblastoma}

Most participants had a child affected by retinoblastoma $(14 / 15,93 \%$, Table 2). Most families were affected by unilateral disease $(9 / 15,60 \%)$. Two participants were survivors themselves; one had an affected child, the other an unaffected child. One of the survivors $(1 / 2,50 \%)$ indicated that they did not undergo genetic testing for retinoblastoma. All affected children $(n=14)$ reportedly underwent genetic testing for retinoblastoma $(14 / 14,100 \%)$. All parents of children with bilateral retinoblastoma $(n=6)$ reported the cancer as being heritable (Fig. 1). Parents of children with unilateral retinoblastoma $(n=8)$ reported the cancer to be, heritable $(n=1)$, non-heritable $(n=4)$, and unknown $(n=$ 3; Fig. 1). Participants or their children had received all or part of their care at the Hospital for Sick Children, however, this does not preclude their treatment and/or follow-up at another Canadian center (the latter data were not collected).

\section{Focus group results}

The results of the focus groups are grouped by the three study questions in the framework (i.e., knowledge, experiences, and attitudes). Themes and subthemes follow for each aspect of the study framework (Table 3).

\section{Knowledge of retinoblastoma genetics}

Three themes were identified in the discussions aiming to elucidate participant knowledge of genetics (Table 3). First, 
Table 2 Study participant experience with retinoblastoma

\begin{tabular}{lll}
\hline & $n$ & $\%$ \\
\hline $\begin{array}{l}\text { Retinoblastoma } \\
\text { (RB) experience }\end{array}$ & & \\
$\quad$ Survivor & 1 & $7 \%$ \\
$\quad$ Mother of child \\
with RB \\
$\quad$ Father of child \\
$\begin{array}{l}\text { with RB } \\
\text { Survivor + } \\
\text { mother of child with }\end{array}$ & 11 & $73 \%$ \\
RB & 2 & $13 \%$ \\
$\quad$ Survivor + father & & \\
of child with RB & 0 & $0 \%$ \\
No. children with \\
retinoblastoma \\
0
\end{tabular}

the participants held the general understanding that retinoblastoma is a genetic disease. However, a second theme revealed that concepts related to retinoblastoma genetics are often miscommunicated or misunderstood. The third theme revealed that participants question the role of the environment and plain 'bad luck' in causing of retinoblastoma.

\section{Retinoblastoma is understood to be a genetic disease}

Generally, participants understood that retinoblastoma was a genetic disease, and often cited the involvement of the $R B 1$ gene.

"[Retinoblastoma is caused by] mutation in the $R B 1$ gene." -Participant N3

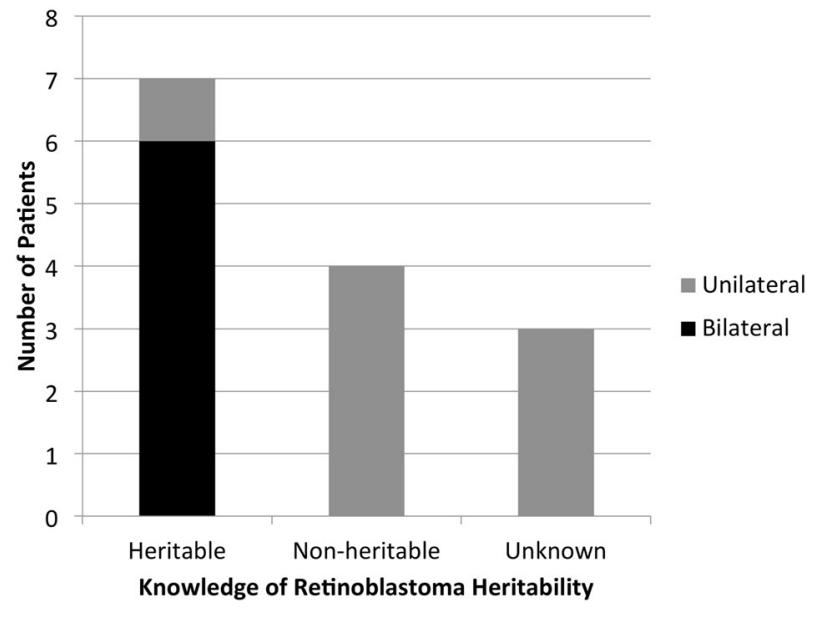

Fig. 1 Genetic testing completed vs. knowledge of retinoblastoma heritability.Of the participants' children, 14 were reportedly diagnosed with retinoblastoma and had undergone genetic testing. All children with bilateral retinoblastoma $(n=6)$ were reported by their parents as being heritable. Of the children with unilateral retinoblastoma $(n=8)$, one was reportedly heritable, four non-heritable, and three unknown

"It's a genetic form of eye cancer."-Participant N4

Some participants attempted more complex explanations of the biological underpinnings of cancer development:

"It's an error during, I'm going to say, mitosis or meiosis, one of the two of them, in the DNA, a piece getting lost and not copied properly. And my understanding is that that piece of DNA on the 13th chromosome is a protein, which would tell the cells to stop growing. Because it's missing, the cells continue to grow." -Participant N4

Genetic concepts are miscommunicated and misunderstood

Conflation between terms 'genetic', 'heritable' and 'inherited: The discussions revealed that individuals generally understood that the term 'genetic' referred to something 'related to DNA', and 'inherited' referred to something derived from one's parents. However, the terms 'heritable' and 'inherited' were often used interchangeably, contributing to confusion about the heritability of retinoblastoma. For example:

"I just look at the genetic and the word heritable as too different like... When I think about genetic, I'm thinking of DNA and strands and when I think of heritable, I'm thinking of y'know just anything that's traditionally passed on through generations of just any sort of disease or condition or you know you have brown hair because your parents have brown hair you know or you go bald because your dad was bald. And I I I just don't think of, I 


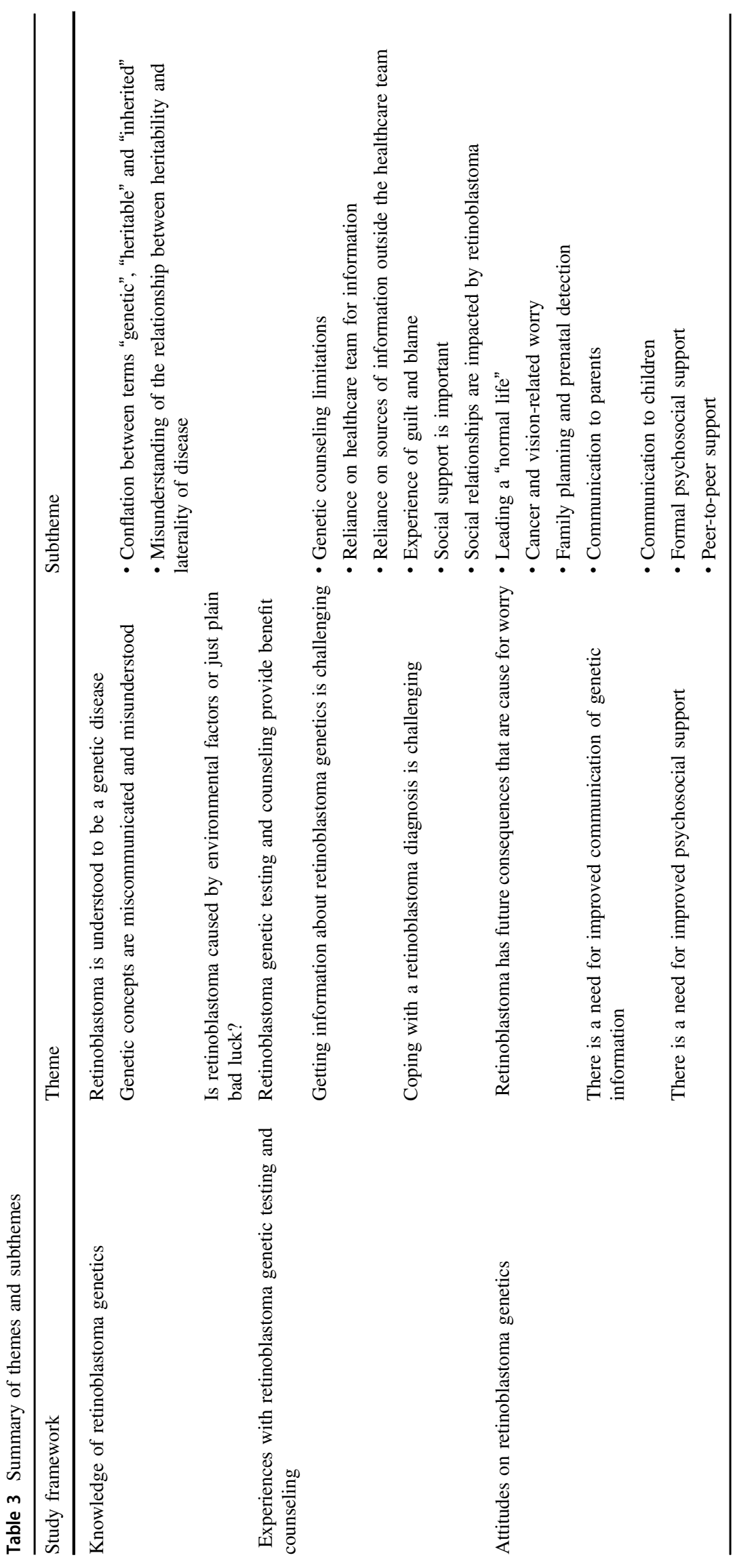


still think it's just some sort of DNA something went that shouldn't have gone. So, I don't know, I always sort of look at that as a two different things because I was tested because I was tested and I don't have the gene, my wife was tested she doesn't have the gene. So I guess I'd, probably feel differently if, one of us did have the gene" Participant N1

Although the participant begins by clarifying the difference between "genetic" and "heritable", the participant concluded their child could not possibly have the heritable form of retinoblastoma, as genetic testing showed it was not inherited from either parent. In actuality, heritable retinoblastoma is most often not inherited from a parent (only 5-10\% of cases are familial), instead a de novo constitutional mutation, which can be passed on to the next generation.

Sometimes, even where participants had articulated the difference between "genetic" and "inherited", the term "genetic form" was used in reference to familial or heritable cases. For example:

"I would just always add that it's a genetic form of eye cancer because [pause] to us it didn't end. And I'm now going through it with my grandchildren." Participant N4

Finally, participants often interpreted the words "mutation" and "genetic" (as a proxy for heritable) to have negative connotations:

"But I would say that while waiting for that diagnosis - that genetic word was the albatross in the room. I will go back to that word because it was like 'please don't let it be genetic' because then we gotta get everybody tested, and it could affect everybody, and it was this horrible big thing... So yeah, genetic was a bad word." -Participant O4

"But I'd still look at it as, mutation. I don't think I don't even like using that word because, what pop culture puts that word into." -Participant N1

Misunderstanding of the relationship between heritability and laterality of disease: The fact that unilateral patients could have the heritable form of retinoblastoma was often a source of confusion, especially for parents of children with unilateral, non-heritable retinoblastoma. Participants with experience of heritable retinoblastoma were often aware of this misconception, and attempted to clarify for others in the group. Take this discussion for example:

Participant O4: "Can I ask a question, just cuz I'm still fairly new to this whole thing. Both you had experience, would it have been bilateral then?"
Participant O1: "Yes."

Participant O3: "Yes."

Participant O4: "Okay, cuz [my daughter] had unilateral”. (Appears relieved).

Participant O5: "So it's not hereditary?"

Participant O4: "It's not hereditary. That's what they say..."

Participant O1: "I think there is also a misconception about that because, just because there is the $50 \%$ chance of passing it on, you're not necessarily going to get it in both eyes. So you can still be considered unilateral, but you still have the DNA mutation."

Participant O3: "Yeah because my ex-husband had one eye affected, but has the genetic form."

Here, Participants $\mathrm{O} 3$ and $\mathrm{O} 1$ recognize that Participant O4 has equated unilateral retinoblastoma with non-heritable retinoblastoma, and attempt to correct that misconception. Through the discussion, Participant O5 also learns that unilateral retinoblastoma can be heritable in some cases. However, by the end of the discussion both Participants $\mathrm{O} 4$ and $\mathrm{O} 5$ turn the discussion away from potential heritability of disease, to inheritance of the disease from the parent. Much later on in the discussion, it becomes clear that Participants $\mathrm{O} 5$ and $\mathrm{O} 4$ are still unclear about the potential for unilateral retinoblastoma to carry a constitutional $R B 1$ mutation:

Participant O5: "My belief, is that with the unilateral - correct [me] if I'm wrong-but is that the secondary cancer risk is not there with the unilateral."

\section{Participant O4: “Don't know.”}

\section{Participant O1: "As long as there's no mutation."}

This discussion is also riddled with imprecise usage of several terms. For example, "DNA mutation" or "mutation" is used in place of "constitutional" or "germline"; technically, all retinoblastoma has mutations. The term "hereditary" is also imprecise, as it does not distinguish between inherited cases (e.g., familial) and those that are new constitutional mutations, which are heritable in the following generation. Amidst this imprecision, the participants fell back to what they felt they understood: since their child's retinoblastoma had not been inherited, this meant that it was not "heritable". 
Is retinoblastoma caused by environmental factors or just plain bad luck?

Some participants expressed the belief that retinoblastoma could be environmentally caused:

"What caused it? Is it because she had a glass of wine before she knew she was pregnant? Or is it because we went on a flight and there was radiation in the sky? ... all these environmental factors that cause cancer you know...really everything seems to cause cancer. Living in a major city, you know, there are 20 different ways it causes, creates, cancer in you, it seems these days. It's the kind of Coke you drink, the air I breathe, the, everything. So I just wonder if something's related to something that caused it." -Participant N1

“Deep down I think it's environmentally caused. Like I think that something in the environment at that point in time." -Participant $\mathrm{O} 2$

Others associated the disease-causing mutation to simply be an unlucky event:

"I don't know and you can just say bad luck or bad genes or bad karma, who knows right? I don't know."

- Participant O5

"I don't know-luck of the draw, pretty much. Just like any other genetic, or not even necessarily genetic. It's just a cell that decided it didn't want to play well with others." -Participant $\mathrm{O} 1$

\section{Experiences with retinoblastoma genetic testing and counseling}

As participants talked about their experiences with genetic testing and counseling for retinoblastoma, three main themes emerged (Table 3). First, genetic testing and counseling was of benefit to the participant. Second, participants identified several challenges in accessing information on retinoblastoma genetics. Third, participants discussed challenges in coping with the retinoblastoma diagnosis.

\section{Retinoblastoma genetic testing and counseling provide benefit}

Participants with multiple generations affected commented on the ability to predict the cancer early with genetic testing:

"So now both of my grandchildren, they're able to find mutations because of the molecular testing. So it's been a big difference for us, from when my daughter was diagnosed "till now." -Participant N4

Genetic testing helped allay some of the guilt for parents who were deemed not to be $R B 1$ mutation carriers:
"Well I sort of had a cataract in my eye. So I thought it might have come from me. I didn't know any better so I just thought about it but... then I asked the doctor and she said 'no'. She just explained the whole thing to me." -Participant D3

Genetic testing also served to confirm somatic cases and eliminate risk of cancer in the unaffected eye:

"With the genetic testing my biggest fear was that it would be affecting the other eye. So with the genetic testing that was at least a relief off of my shoulders just to think 'OK, at least she has her other eye'." -Participant D4

\section{Getting information about retinoblastoma genetics is challenging}

Genetic counseling limitations: Participants who were 'new' to retinoblastoma (non-familial) indicated that genetic information tended to come at a very stressful time during diagnosis. As a result, the participants indicating not retaining much of the information:

"It was just part of the team coming by and social workers and genetics and all that. It was all after surgery. So you know, we sat down...my son's lying in the hospital bed with a patch over his eye and they're trying to tell me about genetics and testing and this and that and honestly I was like 'okay okay', and they told me to sign and I was like 'fine'. I wasn't even paying attention!”-Participant N1

"We definitely did [undergo genetic counseling], right in the height of it all. It's all a blur, but we definitely did." -Participant O5

"I was given two pieces of very devastating news in a very short time period. So I was first told that my son has a-this Chromosome 13 deletion-and I was told at the time that there's not much known about it." -Participant N3

Reliance on healthcare team for information: Some participants recalled receiving resources from members of the healthcare team:

"The genetics team gave us a sheet of a very simplified version of how the two different [forms of retinoblastoma] - the hereditary vs. spontaneous-on the day of the surgery." -Participant D1

One participant cited receiving retinoblastoma genetics information via meaningful engagement with the healthcare team, and this was an individual who had a child with retinoblastoma diagnosed over 30 years ago. 
"I learned a lot through the [healthcare] team. Because I was part of the team for a while. And I went to a couple of World Retinoblastoma Conferences that-I went to Tanzania with the team for the conference." -Participant N4

Reliance on sources of information outside the healthcare team: Participants with a solid grasp of biology due to their academic training mentioned they sought information on retinoblastoma from "university research sites and ... academic journal[s]". However, the vast majority of participants cited social media and general search engines as a source of information:

“There's a Facebook Retinoblastoma group that's interesting to talk to, there's such a variance of degrees and results, from chromosomal deletion up to just unilateral, and people dealing with a lot of different things, a lot of secondary tumors... I think [World Eye Cancer Hope] is a great group and... online " -Participant N4

"For me, my first encounter with any retinoblastoma information was online." -Participant D1

"We did a lot of research online." -Participant O1

One participant indicated they received information from a neighbor of the same cultural background, who also had a retinoblastoma diagnosis in the family:

"Someone introduced me because he had to go through the same thing... So it's easier for us to get information. My wife had the language barrier so, the mother was explaining it to her." -Participant D3

\section{Coping with a retinoblastoma genetic diagnosis is challen- ging}

Experience of guilt and blame: Some participants expressed feeling guilty or responsible for the development of retinoblastoma in their child. This seemed to come up more by participants who had more than one affected generation in their family:

"I'm going through it with my grandkids now, so... Y'know as a mother you carry that guilt, you think you did something wrong because the tumors started in your womb. My daughter was born with those tumors. And uh... now we're back at it with my grandchildren." -Participant N4

"I think my ex-husband felt the same way. He had only one eye affected, so he lived with that. And thought that he wasn't gonna pass it on to his kids. And then when our daughter was born and she was fine. Again, then when my son was born, it was all about the guilt, that it was his fault. He's worried. He would be worried about his son for the next 100 years, because it's his fault." -Participant O3

One example of guilt was expressed through the extended family:

"Because I know that when we were first diagnosed, both my parents, when they heard genetic, they called all of their siblings and asked for everyone's history. They're looking for the root cause. Where did this come from, and my dad found out about a sibling's cousin, someone in the genetic pool that lost an eye, and he was devastated. Because he was like, 'This came from me, I caused this'. And it turned out to be nothing of the sort, but I think when you say genetic, that's the first thing that comes to mind, is "Who's responsible for this?" -Participant O5

Social support is important: Participants indicated that family was often a source of emotional support in coping with retinoblastoma:

"My big thing about going through it all, is that I have a huge family support system, and I'm in the city. And I would not have survived it as well as I did, without my family, my community, everyone coming to support me." -Participant O5

However, some indicated they did not have individuals to turn to for emotional support:

"My biggest challenge was since we didn't tell many people. I had no one to turn to." -Participant N1

Social support was noted as an important factor to have access to and knowledge of early in the diagnosis:

"Just knowing more about where to go for support. I didn't even know about the Facebook [group] until 6 months to a year [after diagnosis]." -Participant N1

Social relationships are impacted by retinoblastoma: Participants felt that relationships with family and friends were impacted by the retinoblastoma diagnosis:

"People are so, they don't know what to say, and they're uncomfortable with the situation." -Participant N2

"It's not easy on the siblings." -Participant N4

They also suggested that they could be assisted greatly by support to communicate with extended family about retinoblastoma genetics:

"I think what could be helpful is if the hospital offered some sort of family counseling...in my family there's a lot of 'through the grapevine', and 
information can get distorted. So it would have been really helpful for me to have a counselor in the room with my [extended family] to just explain to everyone what was going on. As opposed to me having to tell all of them over and over and over, and reliving it."-Participant O5

\section{Attitudes on retinoblastoma genetics}

Three themes were identified in terms of participant attitudes toward genetics of retinoblastoma (Table 3). First, the future consequences of retinoblastoma were perceived to be a source of constant worry. Second, participants expressed a need for improved communications about genetics information to families and children. Third, a request for enhanced psychosocial support was highlighted.

\section{Retinoblastoma has future consequences that are a cause for worry}

Leading a 'normal' life: While the study questions were intended to uncover what parents knew about the future cancer risks and heritability of disease by the next generation, much of the discussion of future consequences of retinoblastoma centered around the desire for affected children to lead "normal" lives and not to be considered "different". For example:

"I feel the more I make the situation normal, the more he'll have the confidence to be like, "What are you talking about?", to another kid that ever says anything to him to make him feel different." -Participant O5

"Can he do everything like sportswise or like drawing, all that stuff initially. Like will he be able to have a normal childhood?" -Participant D1

"Our whole goal through this process is to make sure that he doesn't feel different. And to normalize everything that he experiences. So, we very simply explained to him that, you know, when you were a baby you had cancer. And the doctors had to take your eye, and that got rid of the cancer. And you're all better now." -Participant O5

Cancer and vision-related worry: Most participants identified the risk of second cancers (associated with the heritable form of retinoblastoma) and vision loss as a source of concern. For example:

"I worry that her chances are a little bit greater than someone who has never had any exposure to cancer or anything." -Participant D4
"Having to watch for these signs and symptoms of these potential secondary cancers." -Participant N3

"And also the vision. If something happens to the other eye, then she will be blind." -Participant D4

Family planning and prenatal detection: Most participants identified family planning as a need for themselves or their children:

"If I'm gonna have another baby are they gonna get this?" -Participant D5

"I think that's just the process of consulting with the [retinoblastoma] team and dusting off the genetic reports and testing and doing re-tests to make sure that they're not the carriers. But for me personally, I'm fine with anything that's non-invasive during a pregnancy and then have the baby checked out before she's born." -Participant N1

The risk of miscarriage associated with amniocentesis (i.e., invasive test during pregnancy) was a concern for some. One participant balanced this risk against her personal choice not to ever terminate an affected child, revealing a misconception that the only purpose of prenatal detection would be to terminate a pregnancy (as opposed to the option of early term delivery for initiation of treatment [9], which is part of the standard of care in Canada [8]):

"My daughter said 'I would never terminate a pregnancy for retinoblastoma. Look at me. I lived through it, I had a great life'. And there are all kinds of risks with each of these tests that are invasive. And there's a chance that you could miscarry as a result of the test. So my daughter said I'm not going to do it, I'll take what we get." -Participant N4

\section{There is a need for improved communication of genetic information}

Communication to parents: Participants suggested changes to timing, format, and complexity of information provided could improve communication. With respect to timing:

"You kind of meet genetics in the beginning in the whirlwind of everything that's happening and then there really is no follow-up. So perhaps, just genetics to touch base with us once a year." -Participant O5

"Communication...tends to be a bit of a problem where you're only given information at certain time frames and you don't know the direction where you're going, or you don't know what's changed." -Participant O5 
Suggested formats for information ranged from tangible educational resources to events where discussions could be held:

"Maybe some kind of flow chart. That starts, 'If you've had this, you will get this, you will get this...' And then you know where you fall in the grand plan." -Participant O3

"I think that having these kind of discussion groups and the Retinoblastoma Family Day, and we came to that. And they went around with the geneticists and the doctors and whatnot, I think it gives you a better understanding." -Participant O1

With respect to complexity of information and timing of communication, one participant noted:

"Specifically, with the genetic stuff, I don't know how much you really need to know at the time. Unless you are our case [familial retinoblastoma], and then we can make decisions on what we wanted to do, or to follow the game plan. But off the hop, I don't think knowing the sequence, and knowing that it was this, and all the numbers at the time are really gonna help anything." -Participant O2

Communication to children: Participants discussed the need for digestible educational resources for their children:

"Just an easy resource available...Here is that little pamphlet resource, whatever that one is, I don't know. And here is your little booklet and if you want in the bookstore, you can buy this for this generation that you can really go through the book and explain to your kid ... maybe a flip-up book?" -Participant O4

They also expressed intentions to empower children to explore retinoblastoma on their own:

"I will tell him as much as I know- learn. He's still young so I'm just gonna take it as it goes. Just the information that I get is just so much; resources, data and information that I've studied up on. Tell him that I know he'll learn the skills of looking it up himself and learning about it themselves." -Participant N1

Finally, participants indicated they rely on the healthcare team to help provide education to their children:

"We, [the oncologist] was in the room with [my child] at that point, I didn't get kicked out until later. So [the oncologist] would speak to all of us as a family and say 'Eat healthy, stay out of the sun', that kind of stuff at each sort of appointment." -Participant N1
There is a need for improved psychosocial support Formal psychosocial support: Participants noted the need for more psychosocial supports for themselves, as caregivers of children affected by retinoblastoma:

"I need someone. I need someone to talk to like-I can't talk to my family about it. They're very traditional and I'm sort of in a position of having to be there for my [child]." -Participant N1

"And potentially it would be, again optimistic to do it a little bit, right after, like not during the first 2 days of craziness, but digestion time, and meeting time, or have that first meeting right away, and you have to, you've got a meeting set up for a follow up, and you can choose, to take it away, or to go. Because by having that [psychology] appointment set vs., 'if you feel like you need [an appointment]'...you don't know what you need at that time. And I think that would have been... maybe helpful, maybe not... I don't know, I've never done it. I know we go through a lot as parents." -Participant $\mathrm{O} 4$

Peer-to-peer support: Participants often noted seeking out support from others in the same situation as themselves.

"I used to sit in that waiting room and look around for other kids with glass eyes, and go up to the parents and say, does your kid have retinoblastoma?" -Participant N4

"Other parents...Other, a few other survivors, adult survivors?" -Participant N3

The idea for creating an informal support network was discussed, and one participant noted that one existed long ago:

"I think support is an issue and what we did 25 years ago, 30 years ago was try to put families in touch with each other via the telephone and [the doctors] and I worked together on this program so that you would, [they] would ask a family: 'Are you willing to be part of a program where we put you in touch with another family?"“ -Participant N4

"And I just personally feel that there needs to be better access to other survivors and other parents of survivors because not everyone has the same community support that I had, and that was critical for me." -Participant O5

"We need another Family Day. That brought a lot of people close together." -Participant N3 
"I don't know if they can start a program again where you can put based on-I think you have to do it based a little bit on geography and on diagnosis." -Participant N4

\section{Discussion}

Research into the genetic origins of retinoblastoma has changed our understanding of the disease. New discoveries offer opportunities for new therapies and improvements in overall quality of life for retinoblastoma survivors [5]. In Canada, retinoblastoma genetic testing and counseling is part of the national standard of care [8], yet not much is known about how these services are implemented and understood by retinoblastoma survivors. Our study explored, among retinoblastoma survivors and the parents of children with retinoblastoma, knowledge of, experiences with, and attitudes about retinoblastoma genetics. The intent was to uncover important information that could strengthen understanding, uptake, and application of retinoblastoma genetic information. The study revealed that knowledge of retinoblastoma genetics is variable, and often limited. Although retinoblastoma survivors and parents of children with retinoblastoma know that retinoblastoma is a genetic disorder, this study suggests that the genetic principles of this disease are misunderstood. The most common misconception was thinking that unilateral retinoblastoma held no future cancer risks or risk of heritability by the next generation. No family history of disease, or genetic testing results showing the trait was not transmitted from parents to child, further strengthened the misunderstanding. Generally, knowledge was confined to one's own experience of the disease. Participants with experience of "simple" cases of retinoblastoma (e.g., unilateral non-heritable) often did not fully understand the complexity and range of the clinical manifestation of the disease, largely because it was not relevant to their own case. Taken together with results showing parents and survivors are increasingly looking to online support groups to acquire and share information, this could theoretically pose a problem. There is a need for individuals to fully understand their own situation, and where it falls into the full retinoblastoma spectrum. The participants also came to this conclusion themselves, suggesting the need for better resources such as flowcharts to help them see where their situation fits in.

Genetic counseling represents an important opportunity for healthcare providers to clarify basic genetic concepts, retinoblastoma genetics, and the specific implications for the survivor. In a previous study of genetic testing for retinoblastoma, only $40 \%$ of those who underwent testing additionally received in-person post-test counseling [10].
Although we did not ask this question in our study, one participant (a survivor with an unaffected child) did not have their child tested (Table 2) and 3 of the 14 who reportedly did, failed to understand the result of the test (Fig. 1), suggesting a need for more effective dissemination of test results. Genetic counselors are encouraged to use literature written in layperson vernacular to convey genetic concepts to survivors and families [11]. Yet even still, our participants requested further, more simplified information, at appropriate times during the treatment and follow-up cycle when they feel they are more likely to understand and internalize the information. Participants suggested development of informative and useable written material, which could first explained by the healthcare providers, then offered to survivors for long-term reference. It has previously been reported that retinoblastoma survivors find it difficult to ask for advice from genetic healthcare providers [12]. At the same time, others have characterized a fundamental mismatch between the information genetic counselors provide and what patients with cancer risk want and need to know [13]. Scheduling more frequent or repetitive counseling may not only enhance understanding of concepts, but also provide a comfortable forum and more frequent opportunity for survivors to pose relevant questions.

One participant cited having worked with the retinoblastoma healthcare team in the past, even attending an international scientific conference, as route to gaining knowledge of retinoblastoma genetics. Interestingly, patient engagement in healthcare research is increasingly being cited as a way to help patients adopt and benefit from new and emerging evidence [14]. To this effect, the newly initiated Canadian Retinoblastoma Patient Engagement Strategy (http://lab.research.sickkids.ca/dimaras/research/ engagement) may be a novel way to improve knowledge among affected individuals and their families. This Canadian strategy involves initiatives to recruit a large and diverse retinoblastoma population and facilitate their involvement in all stages of the research process, including priority setting, study design, and execution, and dissemination of results. Involving the patient community in the research process has proven benefits, such as: increased applicability and credibility of results; a heightened sense of autonomy and self-worth among patients; and more effective translation of research results into clinical practice and public policy $[15,16]$.

Risk associated with heritable cancer is a difficult concept for healthcare professionals to communicate to patients and their families [17-20]. Understandably then, it is arguably more challenging for parents of cancer survivors to explain these concepts to their children or other family members. Indeed, participants in this study requested further assistance in communicating information to their children and extended families. Prior research has also pointed 
to a need for improved assistance for parents by the healthcare team in preparation for communicating retinoblastoma health risks to their children [21]. Our participants suggested a need for different and creative ways for medical teams to educate them on retinoblastoma genetics, such as flip-books geared to children, and genetic counseling sessions for extended family members to attend. There is growing support for creative approaches to disseminating genetics information. The first You Tube channel for patients seen in a clinical genetics department, with videos covering common questions asked in familial cancer susceptibility clinics, was widely viewed and had positive patient feedback [22].

Adult childhood cancer survivors' knowledge, risk perception, and treatment history is frequently inaccurate and imprecise [23-26], thus it is important to identify ways to keep the affected child informed of their case into adulthood. The misunderstandings of the implications of genetics on retinoblastoma phenotype and risk described in this study, comprised largely of parents of children diagnosed with retinoblastoma, underscore a probable contributor to adult cancer survivor misinformation. One important genetic service to utilize is comprehensive reproductive counseling, which remains an integral part of care for adult retinoblastoma survivors in Canada. In a 1980-1983 study, retinoblastoma survivors reported more divorces and fewer pregnancies than the general population [27]. The authors attributed the reduction in pregnancies to concern over the heritability of retinoblastoma, and further predicted that the introduction of molecular diagnostic tools after the $R B 1$ gene was mapped in 1983 would offer survivors more options in family planning and help expand their families [27]. Indeed, the introduction of genetic testing for retinoblastoma facilitates more precise determination of risks and eliminates unnecessary examinations of family members deemed not to carry the $R B 1$ mutation, reducing associated worry and costs [28]. More recently, technologies such as preimplantation diagnosis [29-31] offer opportunities for affected parents to give birth to unaffected children, and antenatal genetic diagnosis coupled with early term delivery of $R B 1$ mutationcarrying infants can facilitate earlier treatment of tumors with less invasive therapies and lead to better visual outcomes [9]. A thorough discussion of available reproductive/ diagnostic services and their implications, as well as what it entails to care for an affected child may enhance understanding and better inform survivors' reproductive decisions. Repeated counseling over time may help keep survivors up to date with available options as they evolve [32].

Parents in our study were pre-occupied with their affected children being "normal", or "normalizing" the experience with cancer. Interestingly, survivors and survivor/parents did not share this view, presumably because it did not factor as an issue in their personal experience with retinoblastoma.
When asked to discuss the long-term implications of retinoblastoma, most unaffected parents discussed the physical: a child's athletic capabilities, their future ability to drive, and the general desire for their children to fit in and reach their full potential. Although the question was intended to stimulate conversation about future cancer risks or heritability of retinoblastoma by the next generation, these were not immediate thoughts of these participants. When they were discussed, it appeared these consequences (e.g., future cancer, family planning risks), were known, but the discussion that ensued was one centered on hope and a desire for their kids to have everything they "should" have. Management and communication of future cancer risk and/or family planning was perceived to be the role of medical team. It is possible that the "desire for normalcy" among participants could be at odds with the accurate and effective communication of important genetic information to their affected children. This is even supported somewhat by the suggestion by one participant that "genetic was a bad word" or another not liking the word "mutation", possibly because these are considered "abnormal". Indeed, the language choice of clinical staff may affect how information is interpreted and at times induce unnecessary worry [33]. Perhaps changing the narrative of heritable cancer can help important health concepts be translated to affected children as they grow older. This also may suggest that the healthcare teams need to step in to bring the focus on second cancer risks and family planning to the forefront, where parents may not always do the same. Particularly as many participants noted a need for psychosocial support for themselves, healthcare teams should be cognizant of potential added stress to the caregivers in relaying this sensitive information to their children.

The psychosocial effects endured by families centered largely in all focus group discussions, suggesting that the healthcare teams could do better at linking families to formal support, or facilitate interaction between individuals with experience of retinoblastoma diagnosis.

Our study represents an attempt to gauge the level of knowledge Canadian adult retinoblastoma survivors have regarding the genetics of their disease, and learn how to better meet their informational needs to facilitate family planning and long-term health management. There are certain limitations of our study, namely the small sample size, and that most of the participants were highly educated, unaffected female parents of children with retinoblastoma. The time since first diagnosis of retinoblastoma in the family also varied among participants, such that some participants experienced different standard of care than the current. However, this qualitative study was not meant to result in a generalization of the knowledge of, experiences with, and attitudes about retinoblastoma among survivors and parents of children with retinoblastoma, but to explore their stories to provide insight into future research or 
enhancements to care. Overall, we find that retinoblastoma survivors and parents feel they may benefit from more frequent and enhanced adult counseling, which directly addresses the genotype-phenotype correlation of constitutional RBI mutation and available reproductive options, and clarifies implications for family members based on the genetic status of the disease. Incorporation of feedback mechanisms to assess ongoing understanding may be a way to test and enhance genetic counseling for retinoblastoma in Canada.

Acknowledgements We thank the study subjects whose participation made this study possible. We also acknowledge the Canadian Retinoblastoma Society and The Hospital for Sick Children Retinoblastoma Team for assisting with participant recruitment. This work was supported by a grant from the Canadian GeneCure Foundation.

\section{Compliance with ethical standards}

Conflict of interest The authors declare that they have no conflict of interest.

\section{References}

1. Schneider KI, Schmidtke J. Patient compliance based on genetic medicine: a literature review. J Community Genet. 2014;5:31-48.

2. Mikkelsen EM, Sunde L, Johansen C, Johnsen SP. Psychosocial consequences of genetic counseling: a population-based follow-up study. Breast J 2009;15:61-8.

3. Knudson AG Jr. Mutation and cancer: statistical study of retinoblastoma. Proc Natl Acad Sci USA 1971;68:820-23.

4. Rushlow DE, Mol BM, Kennett JY, et al. Characterisation of retinoblastomas without RB1 mutations: genomic, gene expression, and clinical studies. Lancet Oncol. 2013;14:327-34.

5. Dimaras H, Corson TW, Cobrinik D, et al. Retinoblastoma. Nat Rev Dis Primers 2015;1:15021.

6. Theriault BL, Dimaras H, Gallie BL, Corson TW. The genomic landscape of retinoblastoma: a review. Clin Exp Ophthalmol. 2014;42:33-52.

7. Rushlow D, Piovesan B, Zhang K, et al. Detection of mosaic RB1 mutations in families with retinoblastoma. Hum Mutat 2009;30:842-51.

8. National Retinoblastoma Strategy Canadian Guidelines for Care. Strategie therapeutique du retinoblastome guide clinique canadien. Can J Ophthalmol. 2009;44 Suppl 2:S1-88.

9. Soliman SE, Dimaras H, Khetan V, et al. Prenatal versus postnatal screening for familial retinoblastoma. Ophthalmology 2016;123:2610-17.

10. Cohen JG, Dryja TP, Davis KB, Diller LR, Li FP. RB1 genetic testing as a clinical service: a follow-up study. Med Pediatr Oncol. 2001;37:372-8.

11. Sutherland JE, Day MA. Genetic counseling and genetic testing in ophthalmology. Curr Opin Ophthalmol. 2009;20:343-50.

12. van Dijk J, Oostrom KJ, Huisman J, et al. Restrictions in daily life after retinoblastoma from the perspective of the survivors. Pediatr Blood Cancer 2010;54:110-5.

13. Joseph G, Pasick RJ, Schillinger D, Luce J, Guerra C, Cheng JK. Information mismatch: cancer risk counseling with diverse underserved patients. J Genet Couns. 2017.
14. Kirwan JR, de Wit M, Frank L, et al. Emerging guidelines for patient engagement in research. Value Health 2017;20:481-6.

15. Canadian Institutes of Health Research. Canada's strategy for patient-oriented research: improving health outcomes through evidence-informed care. 2011:1-40. Accessible at: http://www. cihr-irsc.gc.ca/e/44000.html.

16. Domecq JP, Prutsky G, Elraiyah T, et al. Patient engagement in research: a systematic review. BMC Health Serv Res. 2014;14:89.

17. Fischhoff B. Why (cancer) risk communication can be hard. J Natl Cancer Inst Monogr. 1999;25:7-13.

18. Grimes DA, Snively GR. Patients' understanding of medical risks: implications for genetic counseling. Obstet Gynecol. 1999;93:910-4.

19. Rosas-Blum E, Shirsat P, Leiner M. Communicating genetic information: a difficult challenge for future pediatricians. BMC Med Educ. 2007;7:17

20. Overby CL, Chung WK, Hripcsak G, Kukafka R. Cancer genetic counselor information needs for risk communication: a qualitative evaluation of interview transcripts. J Pers Med. 2013;3 (3):238-250.

21. Clarke SA, Sheppard L, Eiser C. Mothers' explanations of communicating past health and future risks to survivors of childhood cancer. Clin Child Psychol Psychiatry 2008;13:157-70.

22. Jones GE, Singletary JH, Cashmore A, et al. Developing and assessing the utility of a You-Tube based clinical genetics video channel for families affected by inherited tumours. Fam Cancer 2016;15:351-5.

23. Cherven B, Mertens A, Meacham LR, Williamson R, Boring C, Wasilewski-Masker K. Knowledge and risk perception of late effects among childhood cancer survivors and parents before and after visiting a childhood cancer survivor clinic. J Pediatr Oncol Nurs. 2014;31:339-49.

24. Cherven BO, Meacham LR. Information needs of childhood cancer survivors: a case for survivor clinic. Pediatr Blood Cancer 2014;61:189-90.

25. Kadan-Lottick NS, Robison LL, Gurney JG, et al. Childhood cancer survivors' knowledge about their past diagnosis and treatment: Childhood Cancer Survivor Study. JAMA 2002;287:1832-9.

26. Maeda N, Horibe K, Kato K, Kojima S, Tsurusawa M. Survey of childhood cancer survivors who stopped follow-up physician visits. Pediatr Int. 2010;52:806-12.

27. Byrne J, Fears TR, Whitney C, Parry DM. Survival after retinoblastoma: long-term consequences and family history of cancer. Med Pediatr Oncol. 1995;24:160-5.

28. Richter S, Vandezande K, Chen N, et al. Sensitive and efficient detection of RB1 gene mutations enhances care for families with retinoblastoma. Am J Hum Genet. 2003;72:253-69.

29. Dhanjal S, Kakourou G, Mamas T, et al. Preimplantation genetic diagnosis for retinoblastoma predisposition. $\mathrm{Br} \mathrm{J}$ Ophthalmol. 2007;91:1090-91.

30. Dommering CJ, Moll AC, Imhof SM, de Die-Smulders CE, Coonen E. Another liveborn after preimplantation genetic diagnosis for retinoblastoma. Am J Ophthalmol. 2004;138:1088-89.

31. Xu K, Rosenwaks Z, Beaverson K, Cholst I, Veeck L, Abramson DH. Preimplantation genetic diagnosis for retinoblastoma: the first reported liveborn. Am J Ophthalmol. 2004;137:18-23.

32. Dommering CJ, Garvelink MM, Moll AC, et al. Reproductive behavior of individuals with increased risk of having a child with retinoblastoma. Clin Genet. 2012;81:216-23.

33. Abramsky L, Fletcher O. Interpreting information: what is said, what is heard--a questionnaire study of health professionals and members of the public. Prenatal Diagnosis 2002;22:1188-94. 